\title{
Generation of Terahertz Radiation by Large-Aperture Photoconductive Antennas in Condition of a High Level Excitation
}

\author{
V.N. TRUKHIN ${ }^{a, b}$ AND A.S. BUYSKIKH ${ }^{a}$ \\ ${ }^{a}$ A.F. Ioffe Physical Technical Institute, 194021 St. Petersburg, Russia \\ ${ }^{b}$ The National Research University of Information Technologies, Mechanics and Optics \\ 194000 St. Petersburg, Russia
}

\begin{abstract}
Theoretical and experimental results about generation coherent terahertz $(\mathrm{THz})$ radiation in photoconductive medium in condition of a high level excitation are presented. Employing self-consistent analytical approach to the set of non-equilibrium Boltzmann equation for charge carriers and Maxwell equations for electromagnetic radiation we have studied the radiation phenomena in non-equilibrium $\mathrm{e}-\mathrm{h}$ plasma excited in the photoconductive gap of terahertz radiating large-aperture photoconductive antenna by an ultrashort laser pulse. Equally with the effect of the radiation screening, the effect of the non-linear absorption of an optical pump pulse (bleaching of photoconductive medium) was taken into account. It was shown that the effect of nonlinear absorption reconstructs the dynamics of the generation of terahertz radiation and must take into account the effects associated with the spread of the generated wave and the wave of excitation.
\end{abstract}

PACS: $72.30+\mathrm{q}, 41.60 .-\mathrm{m}, 72.20 . \mathrm{Jv}$

\section{Introduction}

The effect associated with $\mathrm{THz}$ field amplitude saturation in condition of the increasing pump intensity was observed experimentally in several papers $[1,2]$. There were some explanations of this effect. The first one was the radiation screening mechanism connected with decrease of local field due to $\mathrm{THz}$ field contribution [1]. Another one was the polarization mechanism associated with the fact that the field generated by relocation of excited charge carriers, screens the electronic field applied to large-aperture photocondutive antenna (LPA) [2]. Apparently, the relocation of charge carriers does not take place in the homogeneous photoconductive plate at a uniform excitation. However, there was no mention of non-linear absorption of the excitation pulse at high intensity which decreases the absorption coefficient due to filling of electronic states by excited photocarriers.

This work is aimed at comparing the corrections in the dependence between the amplitude of $\mathrm{THz}$ field and the intensity of the optical pumping. These corrections are associated with the radiation screening and nonlinear absorption of the excitation pulse.

\section{Theoretical considerations}

\subsection{Equations for the field and charge carriers without corrections}

Let us choose a slab geometry in accord with the article [3], in which we investigated the generation of $\mathrm{THz}$ radiation by LPA in condition of a low level excitation (Fig. 1). From system of the Maxwell equations we obtain the following equation for the Fourier component of electric field:

$$
\frac{\mathrm{d}^{2} E_{\omega}}{\mathrm{d} y^{2}}+\frac{\omega^{2}}{c^{2}} E_{\omega}=-\mathrm{i} \omega \mu_{0} J_{z}^{\omega}
$$

where $c=c_{0} / n$ is velocity of light in the medium, $c_{0}$ is velocity of light in free space, $n=\sqrt{\varepsilon \mu}$ is refraction index, $\varepsilon$ and $\mu$ are relative permittivity and relative permeability for the semiconductor on the frequency of generated radiation, $J_{z}^{\omega}$ is $z$ component of the Fourier transform of current and $\mu_{0}$ is vacuum permeability.

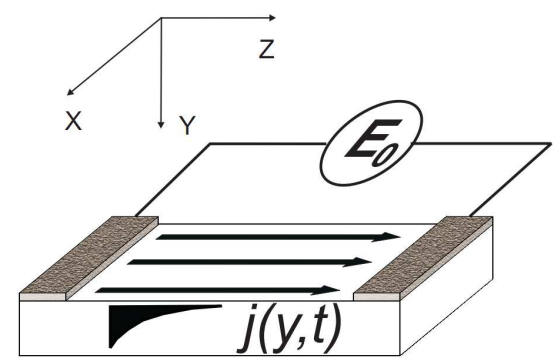

Fig. 1. The sketch of biased semiconductor slab with bias field. Optical pump wave normally illuminates the slab. The black segment shows $y$ distribution of current $j(t, y)$ inside the slab.

The photocurrent density $j(t, y)$ in non-equilibrium e-h plasma created in semiconducting slab biased with an external dc electric field $E_{0}$ is given by [4]: 


$$
\begin{gathered}
J_{z}(t, y)=\frac{\hbar e}{4 \pi^{3}} \int k_{z}\left(f_{\mathrm{ae}}(\boldsymbol{k}, \boldsymbol{r}, t) / m_{\mathrm{e}}\right. \\
\left.-f_{\mathrm{ah}}(\boldsymbol{k}, \boldsymbol{r}, t) / m_{\mathrm{h}}\right) \mathrm{d} \boldsymbol{k} \\
\approx \frac{\hbar e}{4 \pi^{3}} \int k_{z} f_{\mathrm{ae}}(\boldsymbol{k}, \boldsymbol{r}, t) / m_{\mathrm{e}} \mathrm{d} \boldsymbol{k},
\end{gathered}
$$

where $f_{\text {ae }}$ and $f_{\text {ah }}$ are the asymmetric terms of the total distribution functions for electrons, $f_{\mathrm{e}}$, and holes, $f_{\mathrm{h}}$, respectively, $\boldsymbol{k}$ is the electron wave vector, $m_{\mathrm{e}}$ and $m_{\mathrm{h}}$ are the effective masses of free carriers, electrons, and holes, respectively. We can neglect the second term in the right-hand side in compliance with the relationship $m_{\mathrm{e}} \ll m_{\mathrm{h}}$.

When the intensity of optical pump is high enough and the screen effect appears, the concentration of photoelectrons is so high that the time of electron-electron collision becomes less than the energy relaxation time and the impulse relaxation time. Under these conditions we derive the following equations from the Boltzmann equation for electron distribution (projections have chosen in accord with Fig. 1) [5]:

$$
\begin{aligned}
& \frac{\partial n_{\mathrm{e}}}{\partial t}=-\gamma n_{\mathrm{e}}+G(t, y), \\
& \frac{\partial J_{z}}{\partial t}=\frac{e^{2} n_{\mathrm{e}}}{m_{\mathrm{e}}} E_{z}-(\Gamma+\gamma) J_{z},
\end{aligned}
$$

where

$$
\begin{aligned}
& G(t, y)=\frac{\alpha I(t, y)}{\hbar \omega_{0}} \\
& \quad=\frac{\alpha(1-R) I_{0}}{\hbar \omega_{0}} \exp \left(-\alpha y-\frac{\left(t-y / v_{\mathrm{g} 0}\right)^{2}}{2 \tau_{0}^{2}}\right)
\end{aligned}
$$

is the generation term associated with propagation of the Gaussian beam pump on conditions that linear absorption, $n_{\mathrm{e}}, m_{\mathrm{e}}$ are electron density and effective mass, respectively. $\Gamma^{-1}$ is the impulse relaxation time of electrons, $\gamma^{-1}$ is the lifetime of electrons, $\alpha$ is absorption coefficient of pump pulse, $R=\left(n_{\text {pump }}-1\right)^{2} /\left(n_{\text {pump }}+1\right)^{2}$ is the reflection coefficient off the slab interface, $n_{\text {pump }}$ is refractive index for pump. $I_{0}, \omega_{0}$ are the peak intensity and the frequency of pump pulse, $v_{\mathrm{g} 0}=c_{0} / n_{\text {pump }}$ is group velocity of pump, $\tau_{0}$ is pulse width $\left(\mathrm{FWHM}=2.3 \tau_{0}\right)$ and $E_{z}=E_{0}+E_{\mathrm{THz}}$ is the total electromagnetic field including the bias field.

Equation (3) can be solved by using the Fourier transform and then the Fourier component of photocurrent obtained from (4) can be written as

$$
\begin{gathered}
J_{z}^{\omega}(\omega, y)=\frac{e^{2}}{m_{\mathrm{e}}(\Gamma+\gamma-\mathrm{i} \omega)}\left[\frac{E_{0} G^{\omega}(\omega, y)}{\gamma-\mathrm{i} \omega}\right. \\
\left.+\frac{1}{\sqrt{2 \pi}} E_{\mathrm{THz}}^{\omega}(\omega, y) \otimes\left(\frac{G^{\omega}(\omega, y)}{\gamma-\mathrm{i} \omega}\right)\right],
\end{gathered}
$$

where

$$
G^{\omega}(\omega, y)=\frac{\alpha(1-R) I_{0} \tau_{0}}{\hbar \omega_{0}} \exp \left(-\frac{\omega^{2} \tau_{0}^{2}}{2}+\mathrm{i} \frac{\omega}{\tilde{v}_{\mathrm{g}}} y\right)
$$

and

$$
\frac{1}{\tilde{v}_{\mathrm{g}}}=\frac{1}{v_{\mathrm{g} 0}}+\mathrm{i} \frac{\alpha}{\omega}
$$

is the complex group velocity. The second term on the right-hand side of this equation is small correction and we can neglect it in the first approximation. From (5) and (1) we derive the wave equation. Let us consider the simplest case. The medium outside slab is immersion and there are no reflected pulses. While performing a joining of electric field tangential components at the LPA boundaries we get $\mathrm{THz}$ field distribution in the first approximation. The field distribution on the back aperture of the slab producing forward emitting $\mathrm{THz}$ electromagnetic radiation takes the form of

$$
\begin{aligned}
& E_{\mathrm{THz}}^{\omega, 1}(\omega, d+)=-\frac{\tilde{\Xi}(\omega)}{1-\mathrm{i} \omega \tau_{\delta n}} \\
& \quad \times\left[\exp \left(\mathrm{i} \frac{\omega}{c} d\right)-\exp \left(\mathrm{i} \frac{\omega}{v_{\mathrm{g} 0}} d-\alpha d\right)\right],
\end{aligned}
$$

where $\tilde{\Xi}(\omega)=\mu_{0} \frac{e^{2}}{2 \pi^{2} m_{\mathrm{e}}} \frac{\tau_{\mathrm{R}} \tau E_{0}(1-R) I_{0} \tau_{0} c}{\left(1-\mathrm{i} \omega \tau_{\mathrm{R}}\right)(1-\mathrm{i} \omega \tau) \hbar \omega_{0}} \exp \left(-\frac{\omega^{2} \tau_{0}^{2}}{2}\right)$, $\tau_{\delta n}=\alpha^{-1}\left(v_{\mathrm{g} 0}^{-1}-c^{-1}\right), \tau_{\mathrm{R}}=(\gamma+\Gamma)^{-1}, \tau=\gamma^{-1}$. From Eq. (6) we can see that the field is generated at the boundaries of the slab and in the first approximation the field depends on pump intensity linearly. Moreover, due to the superposition it is possible to add as well as subtract the fields. It depends on the phase synchronism between the generated $\mathrm{THz}$ wave and the optical pump wave and also the thickness of plate itself has its part to play.

\subsection{The derivation of corrections}

The $\mathrm{THz}$ output power saturates at high optical pump power due to the decrease of the bias field by the generated $\mathrm{THz}$ field. We solved Eq. (5) by the method of successive approximations. The field obtained in the first approximation was substituted in the second term on the right-hand side of this equation. Further we again solved Eqs. (1) and (5) and joined electric field tangential components at the LPA boundaries. After all these operations we get $\mathrm{THz}$ field distribution in the second approximation. The correction associated with radiation screening can be written as

$$
\begin{aligned}
& E_{\mathrm{THz}}^{\omega, 2}(\omega, d+)=F_{1}(\omega) \exp \left(\mathrm{i} \frac{\omega}{c} d\right) \\
& \quad \times\left[\exp \left(-\alpha d+\mathrm{i} \frac{\omega d}{2 v_{\mathrm{g} 0}}-\mathrm{i} \frac{\omega d}{2 c}\right)-1\right] \\
& \quad+F_{2}(\omega) \exp (-\alpha d) \\
& \quad \times\left[\exp \left(\mathrm{i} \frac{\omega d}{2 v_{\mathrm{g} 0}}+\mathrm{i} \frac{3 \omega d}{2 c}\right)-\exp \left(-\alpha d+\mathrm{i} \frac{\omega d}{v_{\mathrm{g} 0}}\right)\right]
\end{aligned}
$$

where $F_{1}(\omega)=\frac{A \exp \left(-\omega^{2} \tau_{0}^{2} / 4\right)}{(1-\mathrm{i} \omega \tau / 2)\left(1-\mathrm{i} \omega \tau_{R} / 2\right)\left(1-\mathrm{i} \omega \tau_{R}\right)\left(1-\mathrm{i} \omega \tau_{\delta n} / 2\right)^{2}}$, $F_{2}(\omega)=\frac{A \exp \left(-\omega^{2} \tau_{0}^{2} / 4\right)}{(1-\mathrm{i} \omega \tau / 2)\left(1-\mathrm{i} \omega \tau_{3}\right)\left(1-\mathrm{i} \omega \tau_{N} / 2\right)\left(1-\mathrm{i} \omega \tau_{R} / 2\right)\left(1-\mathrm{i} \omega \tau_{R}\right)}$, $A=-\sqrt{\frac{\pi}{2}} \frac{e^{4} c^{2} E_{0} I_{0}^{2}(1-R)^{2} \mu_{0}^{2} \tau \tau_{0}^{2} \tau_{R}^{2}}{4 m_{\mathrm{e}}^{2} \omega_{0}^{2} \hbar^{2}}, \tau_{N}=\left(v_{\mathrm{g} 0}^{-1}+c^{-1}\right) / \alpha$, $\tau_{3}=\alpha^{-1}\left(v_{\mathrm{g} 0}^{-1}-3 c^{-1}\right) / 2$. Equation (7) was obtained with conditions $\omega \tau_{\delta n}<\omega \tau_{N}<1$ and $\omega \tau_{R}<1$. 
The correction associated with the non-linear absorption of the excitation pulse can be obtained when we let the next relation

$$
\alpha\left(n_{\mathrm{e}}\right)=\alpha_{0}\left(1-n_{\mathrm{e}} / n_{0}\right),
$$

where $n_{0}$ is the ultimate concentration. In equation for absorption coefficient (8) in the first approximation electron density has been expressed from Eq. (3). From the Maxwell equations we can get formula for the Gaussian beam pump pulse intensity $\tilde{I}(t, y)$ in case of non-linear absorption. Then Eq. (3) takes the form

$$
\frac{\partial \tilde{n}_{\mathrm{e}}}{\partial t}=-\gamma \tilde{n}_{\mathrm{e}}+\tilde{G}(t, y)
$$

where

$$
\begin{aligned}
& \tilde{G}(t, y)=\frac{\alpha\left(n_{\mathrm{e}}\right) \tilde{I}(t, y)}{\hbar \omega_{0}}=G(t, y)\left\{1+B\left(1-2 \mathrm{e}^{-\alpha_{0} y}\right)\right. \\
& \left.\times \mathrm{e}^{-\alpha_{0} y-\gamma\left(t-y / v_{\mathrm{g} 0}\right)}\left[1+\operatorname{erf}\left(\frac{t-y / v_{\mathrm{g} 0}}{\sqrt{2} \tau_{0}}\right)\right]\right\},
\end{aligned}
$$

$B$ is a positive constant, which is proportional to $I_{0}$. Then by analogy with transformations from Sect. 2.1 we obtained $\mathrm{THz}$ field distribution. We did not take into account radiation screening effect, thus we neglected the second term on the right-hand side of Eq. (5). The correction associated with non-linear absorption can be written as

$$
\begin{aligned}
& \tilde{E}_{\mathrm{THz}}^{\omega, 2}(\omega, d+)=-\Sigma(\omega)\left\{\operatorname { e x p } ( \mathrm { i } \frac { \omega } { c } d ) \left[\frac{1}{1-\mathrm{i} \omega \tau_{\delta n}}\right.\right. \\
& \left.-\frac{1}{1-\mathrm{i} \omega \tau_{\delta n} / 2}\right]-\exp \left(-\alpha_{0} d+\mathrm{i} \frac{\omega}{v_{\mathrm{g} 0}} d\right) \\
& \left.\quad \times\left[\frac{1}{1-\mathrm{i} \omega \tau_{\delta n}}-\frac{\exp \left(-\alpha_{0} d\right)}{1-\mathrm{i} \omega \tau_{\delta n} / 2}\right]\right\},
\end{aligned}
$$

where $\Sigma(\omega)=\frac{e^{2} \mu_{0} E_{0} \alpha_{0} I_{0}^{2}(1-R)^{2} \tau_{0}^{2} c \tau \tau_{R}}{2 \sqrt{2} m_{\mathrm{e}} n_{0} \hbar^{2} \omega_{0}^{2}\left(1-\mathrm{i} \omega \tau_{R}\right)(1-\mathrm{i} \omega \tau)} \Omega(\omega)$ and $\Omega(\omega)=\int_{-\infty}^{+\infty} \exp \left(\sqrt{2} \tau_{0}(\mathrm{i} \omega-\gamma) \theta-\theta^{2}\right)[1+\operatorname{erf}(\theta)] \mathrm{d} \theta$. Apparently, the terms which accord to generated field at the front boundary follow to decrease of total $\mathrm{THz}$ field. The terms which accord to generated field at the back boundary can follow to decrease as well as increase of total $\mathrm{THz}$ field. It depends on the value $\alpha_{0} d$ and a difference between the group velocity of pump $v_{\mathrm{g} 0}$ and the $\mathrm{THz}$ field velocity $c$.

From Eqs. (6), (7) and (10) we estimated the intensity $I_{\mathrm{s}}$ for both effects

$$
I_{\mathrm{s}}(\omega)=\frac{E_{\mathrm{THz}}^{\omega, 1}(\omega, d+) I_{0}}{E_{\mathrm{THz}}^{\omega, 2}(\omega, d+)} .
$$

$I_{\mathrm{s}}$ is the saturation intensity. In this situation the $\mathrm{THz}$ field amplitude diverges from the linear dependence and it can be approximated by the quadratic dependence. Judging by this value we can estimate the value of the pump intensity when the effect decreasing the $\mathrm{THz}$ field in LPA appears. The comparison of the effects of the radiation screening and the non-linear absorption was made for the spectral component for the frequency of $1 \mathrm{THz}$ located near the maximum of the spectral distribution of the generated $\mathrm{THz}$ field.

The calculation was conducted with next parameters: wavelength of the pump wave is $800 \mathrm{~nm}, \mathrm{FWHM}$ is 15 and $100 \mathrm{fs}$, the lifetime of electrons $\gamma^{-1}$ is $580 \mathrm{fs}$ and the impulse relaxation time of electrons $\Gamma^{-1}$ is $40 \mathrm{fs}$. According to the article [6] the value of $n_{0}$ was chosen equal to $2.5 \times 10^{18} \mathrm{~cm}^{-3}$. Also the approximation that $\alpha_{0} d>1$ was taken into account. Hence, the saturation intensity for the radiation screening is $I_{\mathrm{S}}(1 \mathrm{THz})=335 \mathrm{MW} / \mathrm{cm}^{2}$ when FWHM $=100$ fs and $I_{\mathrm{s}}(1 \mathrm{THz})=2.2 \mathrm{GW} / \mathrm{cm}^{2}$ when $\mathrm{FWHM}=15 \mathrm{fs}$. The saturation intensity for the non-linear absorption effect is $I_{\mathrm{S}}(1 \mathrm{THz})=708 \mathrm{MW} / \mathrm{cm}^{2}$ when FWHM $=100$ fs and $I_{\mathrm{S}}(1 \mathrm{THz})=4.7 \mathrm{GW} / \mathrm{cm}^{2}$ when FWHM $=15$ fs. Apparently, the independent estimation indicates that contributions of both effects to the saturation of $\mathrm{THz}$ field are of the same order of magnitude.

\section{Experimental data}

The dependences between the amplitude of $\mathrm{THz}$ field and the intensity of the optical pump were obtained by the pump-probe experiment. LPA consisted of Au strips deposited onto a low temperature (LT)-GaAs layer which has been grown onto GaAs substrate. A distance between Au strips is $300 \mu \mathrm{m}$, and width is $100 \mu \mathrm{m}$. The bias voltage applied to adjacent strips is $100 \mathrm{~V}$. We used femtoseconds Ti:sapphire laser with mean wavelength $800 \mathrm{~nm}$. The experimental dependence of $\mathrm{THz}$ field peak on the pump pulse energy density $J_{\mathrm{s}}$ can be found in Fig. 2. The estimation of $J_{\mathrm{s}}=I_{\mathrm{s}} \mathrm{FWHM}$ is $180 \mu \mathrm{J} / \mathrm{cm}^{2}$, and it coincides with earlier results $190 \mu \mathrm{J} / \mathrm{cm}^{2}$ [1] and $200 \mu \mathrm{J} / \mathrm{cm}^{2}[7]$.

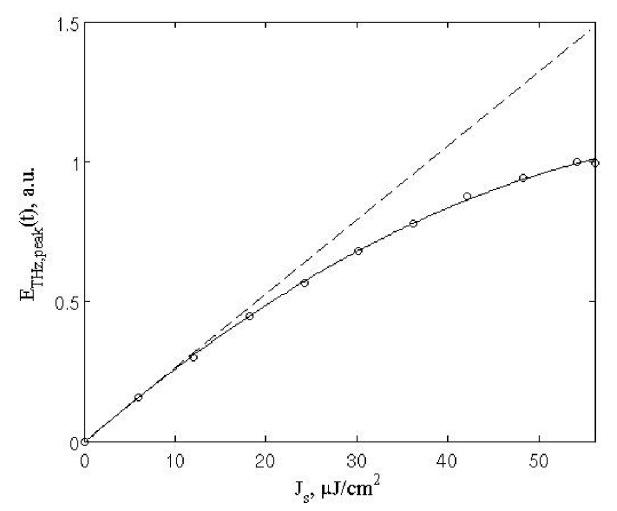

Fig. 2. Experimental data (open circles), quadratic approximation (solid line) and liner approximation (dashed line).

\section{Summary}

As may be seen from the estimated corrections connected with radiation screening and non-linear absorption, the first effect influences a $\mathrm{THz}$ field more than the second effect. Also, calculated value $J_{\mathrm{s}}$ is less than $J_{\mathrm{s}}$ 
obtained from experiments. However, it is necessary to stress that we have made the estimations independently of both effects, whereas in a real situation both of them simultaneously contribute to the amplitude of $\mathrm{THz}$ field. It should not be overlooked that the decease of $\mathrm{THz}$ field at the expense of photocarriers attenuation due to non-linear absorption conducts to the deceasing of radiation effect. Thus, in real situation the value of $I_{\mathrm{S}}$ for radiation screening will be greater. That is why it is necessary to take into account not only non-linear absorption effect, but also the propagation effect of $\mathrm{e}-\mathrm{h}$ plasma. In this condition a local $\mathrm{THz}$ emitter at a back boundary of the photoconductive slab will be taken into account automatically.

In conclusion, we ascertained that the non-linear absorption effect acts important part in the saturation of $\mathrm{THz}$ field in condition of a high excitation. The estimations made on the basis of the paper [6] show that if the pump pulse energy density is $200 \mu \mathrm{J} / \mathrm{cm}^{2}$ then $\mathrm{e}-\mathrm{h}$ plasma with density $2.5 \times 10^{18} \mathrm{~cm}^{-3}$ becomes excited at a depth of $2 \mu \mathrm{m}$ and more. A lot of photoconductive antennas have the thickness of an active generated medium about $1-2 \mu \mathrm{m}$. It made us to come to the conclusion of the necessity (in case of a high level excitation) of taking into account the propagation effect of $\mathrm{e}-\mathrm{h}$ plasma and $\mathrm{THz}$ radiation.

\section{References}

[1] J.T. Darrow, X.-C. Zhang, D.H. Auston, IEEE J. Quantum Electron. 28, 1607 (1992).

[2] M. Tonouchi, N. Kawasaki, T. Yoshimura, H. Wald, P. Seidel, Jpn. J. Appl. Phys. 41, L706 (2002).

[3] V.N. Truchin, A.V. Andrianov, N.N. Ziniv'ev, Phys. Rev. B 78, 155325 (2008).

[4] L.D. Landau, E.M. Lifshitz, in: Physical Kinetics, Eds. Yu.G. Rudnoy, E.V. Morozova, Pergamon, Oxford 1991.

[5] F.G. Bass, Yu.G. Gurevich, in: Hot Electrons and Strong Electromagnetic Waves in Semiconductor Plasmas and Gas-Discharge, Eds. I.G. Virko, L.V. Lihacheva, Nauka, Moscow 1975.

[6] F. Kadlec, H. Nemec, P. Kuzel, Phys. Rev. B 70, 125205 (2004).

[7] G.C. Loata, M.D. Thomson, T. Löffler, H.G. Roskos, Appl. Phys. Lett. 91, 232506 (2007). 